\title{
Dermatitis and systemic mycosis in lined seahorses Hippocampus erectus associated with a marine- adapted Fusarium solani species complex pathogen
}

\author{
Caroline E. Salter ${ }^{1}$, Kerry O'Donnell ${ }^{2}$, Deanna A. Sutton ${ }^{3}$, David P. Marancik ${ }^{1}$, \\ Susan Knowles ${ }^{1}$, Tonya M. Clauss ${ }^{4}$, Aimee L. Berliner ${ }^{4}$, Alvin C. Camus ${ }^{1, *}$ \\ ${ }^{1}$ Department of Pathology, The University of Georgia College of Veterinary Medicine, Athens, Georgia 30602, USA \\ ${ }^{2}$ Bacterial Foodborne Pathogens and Mycology Research Unit, National Center for Agricultural Utilization Research, \\ US Department of Agriculture, Agricultural Research Service, Peoria, Illinois 61604, USA \\ ${ }^{3}$ Department of Pathology, University of Texas Health Science Center, San Antonio, Texas 78229, USA \\ ${ }^{4}$ Department of Veterinary Services, Georgia Aquarium, Inc., Atlanta, Georgia 30313, USA
}

\begin{abstract}
During a 4 mo epizootic, $100 \%$ of 152 lined seahorses Hippocampus erectus in 3 separate groups died while in quarantine following shipment to a public aquarium. Twelve animals with skin depigmentation and ulceration were received by the Aquatic Pathology Service, College of Veterinary Medicine, University of Georgia, Athens, Georgia, USA, for diagnostic evaluation. Microscopically, lesions in 11 seahorses included multifocal epithelial necrosis and ulceration associated with 2 to $7 \mu \mathrm{m}$ diameter, branching, septate fungal hyphae, typically accompanied by deeper infiltration into underlying skeletal muscle. Angioinvasion, with vascular thrombosis and tissue infarction, was a prominent feature in multiple animals. Fungal invasion of one or more internal organs was observed in 4 animals. Hyphae appeared to course freely through tissues and elicited little or no inflammatory response. Fusariosis has been reported sporadically in fish and other aquatic organisms, but identification has often been limited to the genus level based solely on morphologic features. Morphologic characteristics of the fungus isolated from this case were consistent with the Fusarium solani species complex (FSSC), which includes over 50 members that can only be identified definitively using DNA sequence data. A 3-locus typing scheme identified the isolate as a distinct species/haplotype, designated FSSC 12-a, belonging to a specific lineage that appears adapted to aquatic environments and disease in marine animals. Empirical treatment with itraconazole failed to stop mortalities, and subsequent in vitro antifungal susceptibility data explained a lack of clinical efficacy for this agent. Effective treatment in human medicine has similarly been limited by poor susceptibility to several classes of antifungal compounds.
\end{abstract}

KEY WORDS: Seahorse $\cdot$ Fusarium $\cdot$ Mycosis $\cdot$ Histopathology $\cdot$ DNA typing $\cdot$ Phylogenetic analysis

\section{INTRODUCTION}

Fusarium spp. are non-dematiaceous filamentous fungi (Roilides et al. 2007) found in soil, plants, air (Nelson et al. 1994), seawater, and river water (Pal- mero et al. 2009). The genus includes plant pathogens, mycotoxin producers, and opportunistic pathogens of vertebrate hosts immunocompromised by stress or concurrent disease (Nelson et al. 1994, Noga 2010). Reports of fusarial infections include dissemi- 
nated mycosis in immunocompromised human patients (Dignani \& Anaissie 2004), keratitis in horses and humans (Mitchell \& Attleburger 1973, Dignani \& Anaissie 2004, Chang et al. 2006, O'Donnell et al. 2008), dermatitis in toads, sea turtles, and marine mammals (Frasca et al. 1996, Cabañes et al. 1997, Perpiñán et al. 2010), embryonic death in sea turtles (Sarmiento-Ramírez et al. 2010), and black gill disease in cultured Kuruma prawn Penaeus japonicas (Bian \& Egusa 1981) and the giant tiger prawn P. monodon (Khoa et al. 2004). Fusarial infections in freshwater and marine fish include deep mycoses, ocular and skin lesions, and fatal ulceration and necrohemorrhagic dermatitis (Yanong 2003, Noga 2010).

Historically, the morphospecies Fusarium solani has been reported as the etiological agent responsible for most fusarioses in humans and other animals. However, molecular phylogenetic studies have resolved a genealogically exclusive $F$. solani species complex (FSSC), with 3 clades containing at least 45 phylogenetically distinct species (O'Donnell 2000, Zhang et al. 2006, O'Donnell et al. 2008). Members of the FSSC have been implicated in approximately two-thirds of documented cases of fusariosis in humans and animals worldwide. However, vertebrate isolates have only been associated with species in Clade 3, while Clades 1 and 2 exclusively contain plant pathogens (Zhang et al. 2006, O'Donnell et al. 2008). Morphologic similarities between species within the FSSC often confound identification, emphasizing the importance of molecular phylogenetic evaluation for identifying species within this speciesrich complex (O'Donnell et al. 2010).

The lined seahorse Hippocampus erectus is a teleost fish in the family Syngnathidae found in the western Atlantic Ocean from Nova Scotia, Canada, to Argentina (Dias et al. 2002, Ahnesjö \& Craig 2011). Lined seahorses inhabit a range of marine habitats in both shallow and deep water and have been found in association with coral, seagrass beds, mangrove roots, and floating seaweed (Dias et al. 2002). Commercial trade in seahorses for medicinal uses and display in public and private animal collections is a significant global industry. Unfortunately, due to overexploitation and unintentional catch by shrimping operations (Vincent et al. 2011), H. erectus is now listed as Vulnerable to extinction on the IUCN Red List of Threatened Species (IUCN 2011).

Much of the current knowledge regarding disease in syngnathid fishes comes from observations made from morbidities and mortalities in public aquariums and aquaculture facilities. A number of endo- and ectoparasites have been reported and treated successfully, including Cryptobia- and Ichthyobodo-like flagellates, Amyloodinium ocellatum, an Argulus sp., and intestinal nematodes and cestodes. Uronema marinum and the microsporidian Glugea heraldi have also been described in syngnathids but have limited to no treatment options, respectively (Koldewey 2005). Significant diseases include mycobacteriosis and Vibrio harveyi septicemia, and fungal exophilalosis (Blazer \& Wolke 1979, Koldewey 2005, Nyaoke et al. 2009). Herein we report on the gross and histopathologic findings associated with an epizootic of fusariosis in a group of lined seahorses, as well as the molecular identification and phylogenetic characterization of the agent as an unnamed phylogenetic species designated FSSC 12-a Fusarium sp. While FSSC 12-a is known to cause disease in other marine animals, the condition has not been previously described in syngnathid fish.

\section{MATERIALS AND METHODS}

\section{Fish and case history}

Between 30 July and 20 October 2010, 152 juvenile lined seahorses, 3 to $10.2 \mathrm{~cm}$ in overall length, were received into the quarantine facility of a public aquarium. The seahorses were captive bred at 4 separate facilities in the US. Two groups remained segregated after arrival, and 2 groups were combined in a single tank. The fish were housed in acrylic tanks each supplied with recirculating artificial seawater (Instant Ocean ${ }^{\circledR}$ Sea Salt, Spectrum Brands), fluidized bed biofilters, and bio-ball filled sump tanks. Two tanks (Q22, Q23) were located adjacent to each other, with the third (TPO) approximately $5 \mathrm{~m}$ away. Each system was maintained independently, with no shared implements to prevent cross contamination. Feed consisted of live and frozen mysid shrimp offered twice daily. Additional husbandry and clinical information is summarized in Table 1.

During the epizootic, various continuous immersion antibiotic treatments were attempted individually and in succession in different tanks, including: $1 \mathrm{mg} \mathrm{l}^{-1}$ oxolinic acid for $7 \mathrm{~d}, 30 \mathrm{mg} \mathrm{l}^{-1}$ trimethoprim sulfathiazole sodium for 10 to $20 \mathrm{~d}$, or $50 \mathrm{mg} \mathrm{l}^{-1}$ kanamycin sulfate for 10 d (National Fish Pharmaceuticals). Two-hour bath treatments with itraconazole (Sporonox ${ }^{\circledR}$, Centocor Ortho Biotec) were instituted in one group at $1 \mathrm{mg} \mathrm{l}^{-1}$ for $12 \mathrm{~d}$ after histologic evidence of fungal infection was established. 
Table 1. Hippocampus erectus. Summary of husbandry and clinical information in 3 recirculating seahorse culture systems experiencing the Fusarium solani species complex (FSSC) 12-a Fusarium sp. epizootic

\begin{tabular}{|lccccccc|}
\hline System & $\begin{array}{c}\text { Volume } \\
\text { (l) }\end{array}$ & $\begin{array}{c}\text { No. } \\
\text { of } \\
\text { ind. }\end{array}$ & $\begin{array}{c}\text { Arrival } \\
\text { date } \\
(2010)\end{array}$ & \multicolumn{2}{c}{$\begin{array}{c}\text { Time to } \\
\text { mortality (d) }\end{array}$} & $\begin{array}{c}\text { Avg. } \\
\text { temp }\end{array}$ & $\begin{array}{c}\text { Avg. } \\
\text { salinity } \\
\left(\mathrm{mg} \mathrm{l}^{-1}\right)\end{array}$ \\
\hline Q22 & 350 & 32 & 30 July & 9 & 82 & 26.0 & 32 \\
Q23 & 350 & 80 & 11-12 August & 4 & 41 & 26.5 & 33 \\
TPO & 675 & 40 & 20 October & 14 & 53 & 23.5 & 33 \\
\hline
\end{tabular}

\section{Gross necropsy and histopathology}

Over the course of the epizootic, a total of 11 deceased animals were randomly collected for histopathologic examination from the 152 seahorses that died. Their coelomic cavities were incised and bodies fixed in $10 \%$ neutral buffered formalin, followed by submission to the Aquatic Pathology Service, Department of Pathology, University of Georgia College of Veterinary Medicine, Athens, GA, USA. An additional specimen was submitted fresh for fungal culture and histopathology. Direct microscopy of gill clips and skin scrapings was performed using standard methods (Stoskopf 1993). A small tissue wedge was collected from the margin of a skin lesion and submitted for fungal culture. Following fixation, the bodies were decalcified for $24 \mathrm{~h}$ in Kristensen's solution $(3.4 \%$ sodium formate in $4 \mathrm{M}$ formic acid; Kristensen 1948) and then cut into 2 to $3 \mathrm{~mm}$ cross sections. Tissue samples were processed routinely by dehydration in a graded series of ethanol solutions of increasing strength, followed by clearing in a series of xylenes, embedding in paraffin, and sectioning at $5 \mu \mathrm{m}$. Prepared slides were stained with hematoxylin and eosin (H\&E; Allen 1992).

\section{Mycology and bacteriology}

Initial fungal isolation was made on Sabouraud dextrose agar (SDA) plates (Remel) incubated at $22^{\circ} \mathrm{C}$. A subculture of the isolate was submitted to the Fungus Testing Laboratory, Department of Pathology, University of Texas Health Science Center, San Antonio, TX, USA, for species identification. It was accessioned into their culture collection as UTHSC 10-3125 and further subcultured onto potato flake agar (PFA; Rinaldi 1982) and carnation leaf agar (CLA; Nelson et al. 1983), prepared in-house. Macroscopic and microscopic features were determined from cultures incubated at $25^{\circ} \mathrm{C}$ for $7 \mathrm{~d}$ under
$12 \mathrm{~h}$ light and dark cycles. Antifungal susceptibility testing was performed according to the Clinical and Laboratory Standards Institute (CLSI) M38A2 method for filamentous fungi (CLSI 2008).

Samples of liver and kidney, collected aseptically prior to formalin fixation, were received for bacterial and mycobacterial culture from 1 seahorse also submitted for histopathologic examination. Tissues were streaked onto tripticase soy agar with $5 \%$ sheep blood and MacConkey agar plates, as well as LowensteinJensen slants (Remel). Agar plates were incubated aerobically for 24 to $48 \mathrm{~h}$, and Lowenstein-Jensen slants were observed for $45 \mathrm{~d}$ for mycobacterial growth. Plates and slants were incubated at $29^{\circ} \mathrm{C}$. Bacterial identification was made using a Sensititre ARIS 2X and identification plates (TREK Diagnostic Systems) following the manufacturer's instructions.

\section{Molecular identification and phylogenetic analysis}

Based on the morphological identification, a subculture of the fungus was accessioned in the ARS Culture Collection (http://nrrl.ncaur.usda.gov; NCAUR-ARS-USDA, Peoria, IL, USA) as NRRL 54721 Fusarium sp. for DNA sequence-based typing. Published protocols for extraction of total genomic DNA, PCR amplification, and DNA sequencing were followed (Zhang et al. 2006, O'Donnell et al. 2008). In brief, fungal mycelium was harvested from a yeastmalt broth culture and freeze-dried overnight. Total genomic DNA was extracted using hexadecyltrimethyl-ammonium bromide (Sigma) as previously described (O'Donnell et al. 1998). Published PCR primers were used to amplify and sequence a portion of the translation elongation factor $(E F-1 \alpha)$ gene, using an Applied Biosystems (ABI) 3730 capillary sequencer. After the sequence chromatograms were edited and assembled in Sequencher version 4.10.1 (Gene Codes), the corrected sequence was used as a BLAST query of GenBank (http://blast.ncbi.nlm.nih. gov/), Fusarium-ID (http://isolate.fusariumdb.org/; Geiser et al. 2004), and the database Fusarium MLST (www.cbs.knaw.nl/Fusarium/). Because these queries indicated that NRRL 54721 was nested within the FSSC, portions of the second-largest subunit of RNA polymerase (RPB2) and the nuclear ribosomal internal transcribed spacer region (ITS rDNA) and domains D1 and D2 of the nuclear large subunit (LSU 
rDNA) were sequenced to identify the phylogenetic species and 3-locus haplotype as previously described (O'Donnell et al. 2008).

Sequences of NRRL 54721 were edited and exported from Sequencher version 4.10.1 as NEXUS files that were aligned with a comprehensive 3locus dataset for the FSSC (O'Donnell et al. 2008) using MUSCLE (Edgar 2004). Maximum parsimony (MP) analyses were conducted with PAUP* version 4.0b10 (Swofford 2002), and maximum likelihood (ML) analyses were implemented in GARLI (Zwickl 2006). Clade support was assessed via 1000 bootstrapped pseudoreplicates of the data. COLLAPSE version 1.1 was used to identify the FSSC species and 3-locus haplotype of NRRL 54721. Sequences of NRRL 54721 Fusarium sp. FSSC 12-a were deposited in GenBank under accession numbers JQ743207 to JQ743212.

\section{RESULTS}

\section{Gross and histopathologic findings}

Prior to death, some moribund animals were observed with ill-defined areas of depigmentation or with cottony-white patches on the skin. Grossly, 11 of 12 seahorses submitted had variably distributed skin lesions often localized to natural body prominences and the tip of the tail. Lesions were present along the entire body length in 3 animals. In the fresh specimen, irregular, poorly demarcated lesions up to $0.5 \mathrm{~cm}$ in maximum width varied from inconspicuous areas of depigmentation to focal ulcers that exposed the white underlying dermis and were bordered by ragged, loosely adhered margins (Fig. 1A). Wetmount preparations of skin and gills revealed no external parasites. However, septate fungal hyphae with acute angle branching and large, fusiform macroconidia were present in scrapings of skin lesions (Fig. 1B).

Histologically, lesions most commonly involved the skin and vascular system. Organ system involvement was limited to only a small number of cases, as described below. However, there was marked variation in the severity and distribution of lesions between individuals. Hyphae appeared to course through tissues unimpeded, resulting in minimal changes to extensive necrosis. Inflammation was a minor component of the majority of lesions.

Skin changes varied from foci of epithelial necrosis and erosion (Fig. 1C) to transmural ulceration into underlying skeletal muscle. Exposed dermal tissues
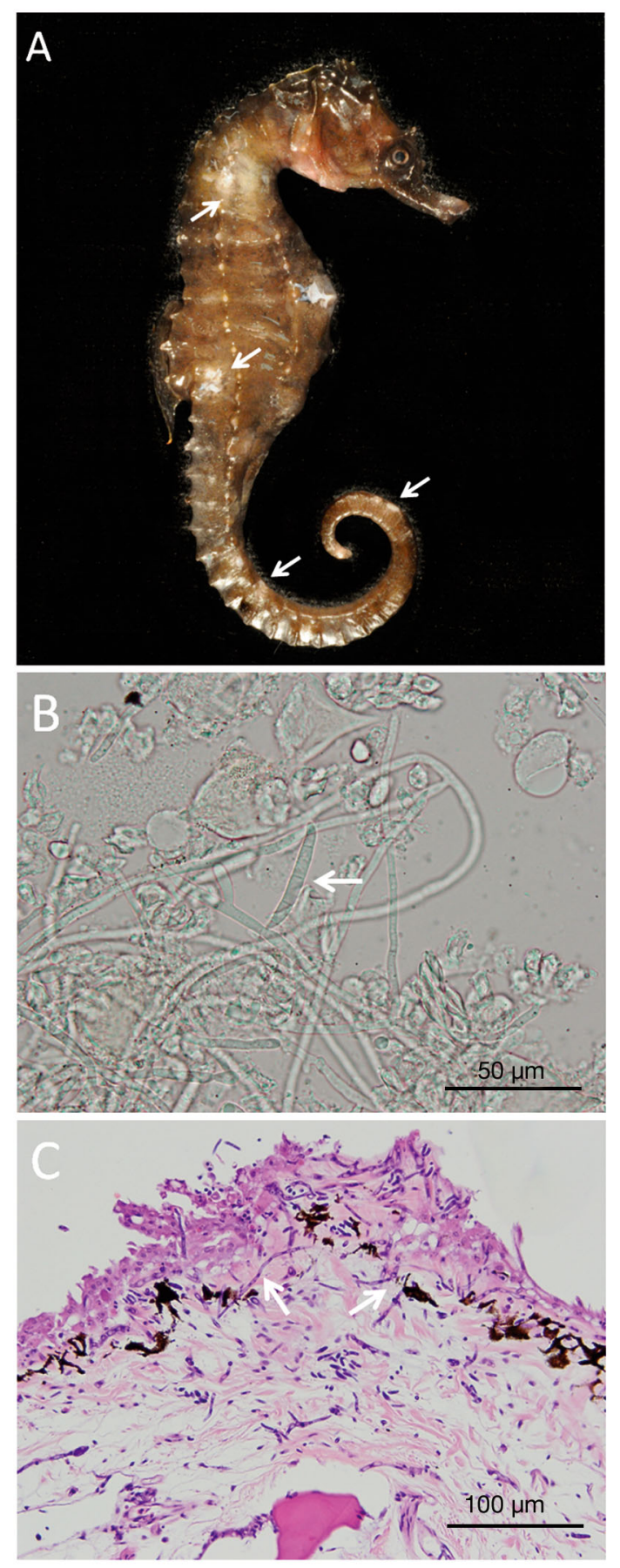

Fig. 1. Fusarium solani species complex (FSSC) infecting Hippocampus erectus. (A) Gross photograph of a lined seahorse infected with FSSC 12-a Fusarium sp. Note multiple foci of poorly demarcated epidermal depigmentation and ulceration of the head, trunk, and tail (arrows). (B) Wet mount of a skin scraping of a gross lesion showing branching septate hyphae and large septate macroconidia (arrow) of FSSC 12-a Fusarium sp. Scale bar $=50 \mu \mathrm{m}$. (C) Micrograph of necrotic $H$. erectus epidermis over a natural body prominence, showing hyphal invasion (arrows) of an edematous dermis with little to no inflammatory response to the presence of hyphae. H\&E stain. Scale bar $=100 \mu \mathrm{m}$ 
were typically necrotic and edematous, as evidenced by loss of tinctorial properties, fibrillation and separation of collagen fibers. Hyphal invasion of skeletal muscle occurred in all seahorses to varying depths. Hyphae infiltrated through interstitial connective tissues and myofibers with no apparent effect, or were associated with necrosis of individual and small groups of muscle fibers, characterized by pale to hypereosinophilic, condensed, and vacuolated sarcoplasm devoid of cross striations. Muscle involvement appeared to result from direct extension of epidermal lesions, but occurred secondarily to vascular invasion in the absence of skin ulceration in at least 1 animal.

Angioinvasion was a prominent feature of lesions in 10 of the 12 cases, involving structures as small as dermal capillaries to large elastic arteries including the dorsal aorta and carotid arteries (Fig. 2). As in muscle, hyphae coursed through vascular walls and lumens, resulting in a spectrum of changes from none to transmural necrosis, thrombosis, and infarction of surrounding perivascular tissues. Hyphae were observed in the gills of 3 animals. No microscopic changes were identified in one individual, while mild lamellar epithelial hypertrophy and hyperplasia with synechia formation was present in a second. Multiple holobranchs were unilaterally thrombosed and destroyed in the third, where an extensive mat of hyphae extended from the necrotic gill tissue to fill the adjacent opercular cavity.

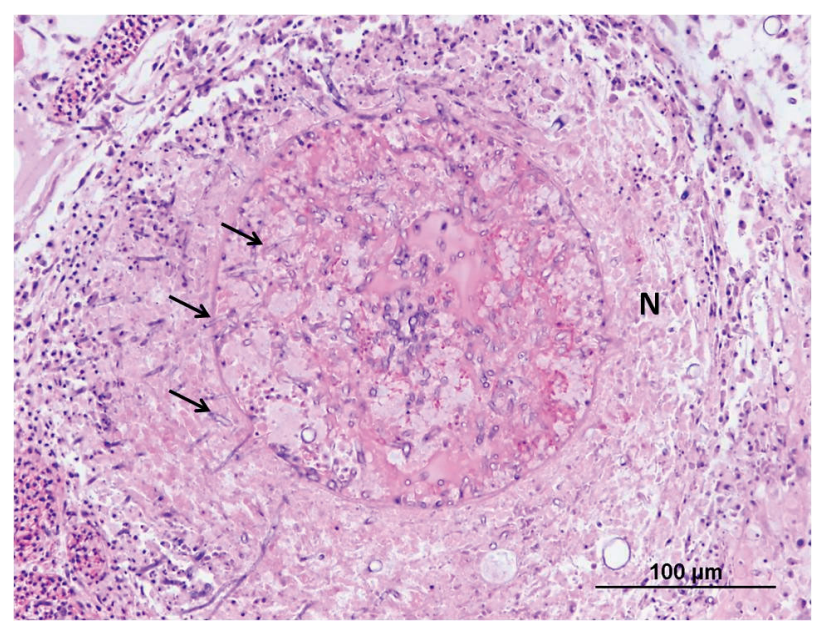

Fig. 2. Fusarium solani species complex (FSSC) infecting Hippocampus erectus. Thrombosis of lined seahorse carotid artery with transmural necrosis $(\mathrm{N})$ of the vascular wall and infarction of adjacent tissue caused by FSSC 12-a Fusarium sp. Hyphae are indicated by arrows. H\&E stain. Scale bar = $100 \mu \mathrm{m}$
Invasion of additional organs and variable necrosis was present in 3 animals. In one seahorse, hyphae were present in the gastrointestinal tract, kidney, and liver. A second animal had diffuse hyphal invasion of the kidney and spinal cord. Rare hyphae were found throughout the coelomic cavity of a third animal.

Hyphae were moderately basophilic with H\&E stains and were readily visible without additional special staining procedures. Hyphae were morphologically similar in all animals, possessing thin, generally parallel walls, 2 to $7 \mu \mathrm{m}$ in diameter, with variably spaced septa, extensive vacuolation, and frequent acute and right angle branching (Fig. 3). Despite the extent of lesions and presence of numerous hyphae, invasion was rarely associated with significant inflammation. Hyphae did not appear to elicit a direct inflammatory cell response. Changes were limited primarily to small numbers of macrophages scattered in infected connective tissues and perivascular sites.

Bacteria were observed by light microscopy or were cultured from 9 of 12 seahorses. In the one animal received fresh and cultured, very light to light growth of Vibrio spp. Group 6 was isolated from the liver and kidney, in addition to light growth of Listonella anguillarum from the kidney. Mycobacterial cultures of these tissues were negative. No bacteria or lesions attributable to bacteria were observed histologically in either tissue. Although bacterial rods were present in the dermis of 4 fish and were distrib-

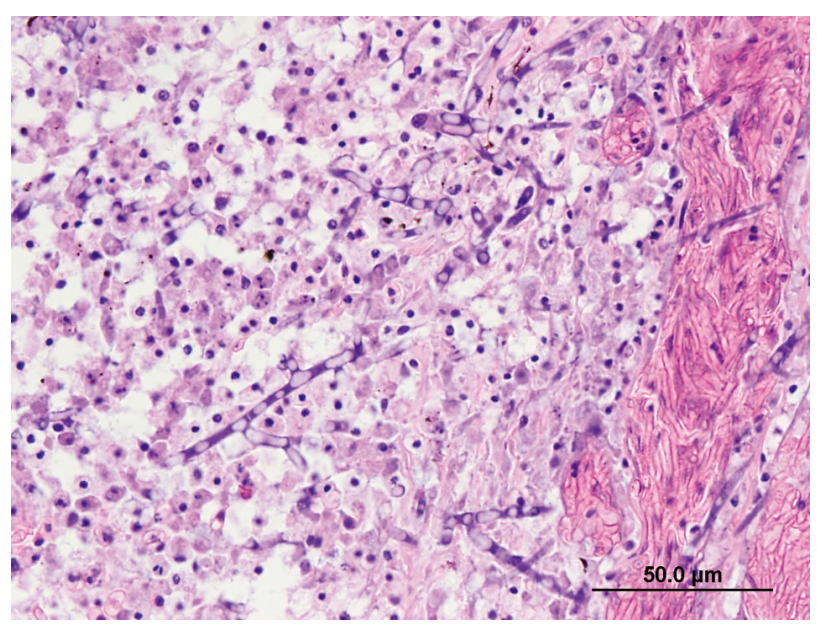

Fig. 3. Fusarium solani species complex (FSSC) infecting Hippocampus erectus. FSSC 12-a Fusarium sp. hyphae among necrotic cellular debris unilaterally filling the opercular cavity of a lined seahorse. Hyphae are septate, extensively vacuolated, 2 to $7 \mu \mathrm{m}$ in diameter with thin, predominantly parallel walls and acute and right angle branching. H\&E stain. Scale bar $=50 \mu \mathrm{m}$ 
uted in multiple organs of an additional 3, their presence did not result in observable tissue changes or inflammation.

\section{Morphological and molecular characterization of the Fusarium mycotic agent}

A fungal isolate tentatively identified as a Fusarium sp. was submitted to the Fungus Testing Laboratory for species identification. The isolate on SDA was wooly, with a rosy, gray-brown obverse and rusty-brown reverse. Colonial growth on PFA was light tan, with a cream-colored reverse. The dominant feature on both PFA and CLA was the production of macroconidia formed in cream-colored sporodochia (masses of conidiophores and macroconidia) that were long, thin, possessed foot cells, and had a distinct curvature (Fig. 4A). A few 1- and 2-celled microconidia were also present at the tips of long monophialides (phialides with a single opening) and were held together in loose balls referred to as 'false heads' (Fig. 4B). Although no chlamydoconidia were observed on CLA after $7 \mathrm{~d}$, other morphologic features were consistent with a member of the FSSC.

The partial $E F-1 \alpha$ gene sequence of the isolate used to query GenBank, Fusarium-ID, and Fusarium MLST revealed that NRRL 54721 might be nested within phylogenetic species FSSC 12 within Clade 3 of the FSSC as defined by O'Donnell et al. (2008). Therefore, partial sequences of RPB2 and the nuclear ribosomal ITS+LSU rDNA were generated so that this isolate could be typed using the 3-locus typing scheme developed to identify phylogenetic species and haplotypes within the FSSC (O'Donnell et al. 2008). A 3-locus dataset was assembled comprising sequences of the 7 known FSSC 12 isolates together with those of 12 closely related FSSC species comprising 25 additional sequences. MP and ML analyses of the 3-locus dataset and the COLLAPSE ver. 1.1. analysis indicated that isolate NRRL 54721 was phylogenetic species/haplotype FSSC 12-a Fusarium sp. (Fig. 5). The isolate shared the same 3-locus haplotype with NRRL 22834 isolated from a western Australian spiny rock lobster Panulirus cygnus with mycosis from Australia and NRRL 22642 from the gills of Kuruma prawn Penaeus japonicus suffering from black gill disease in Japan (O'Donnell et al. 2008). The 8 isolates of phylogenetic species FSSC 12 were all recovered from diverse marine animals or from sand in a marine aquarium (Azor et al. 2007).

Susceptibility testing of isolate NRRL 54721 FSSC 12-a Fusarium sp. to fluconazole, itraconazole, posa-

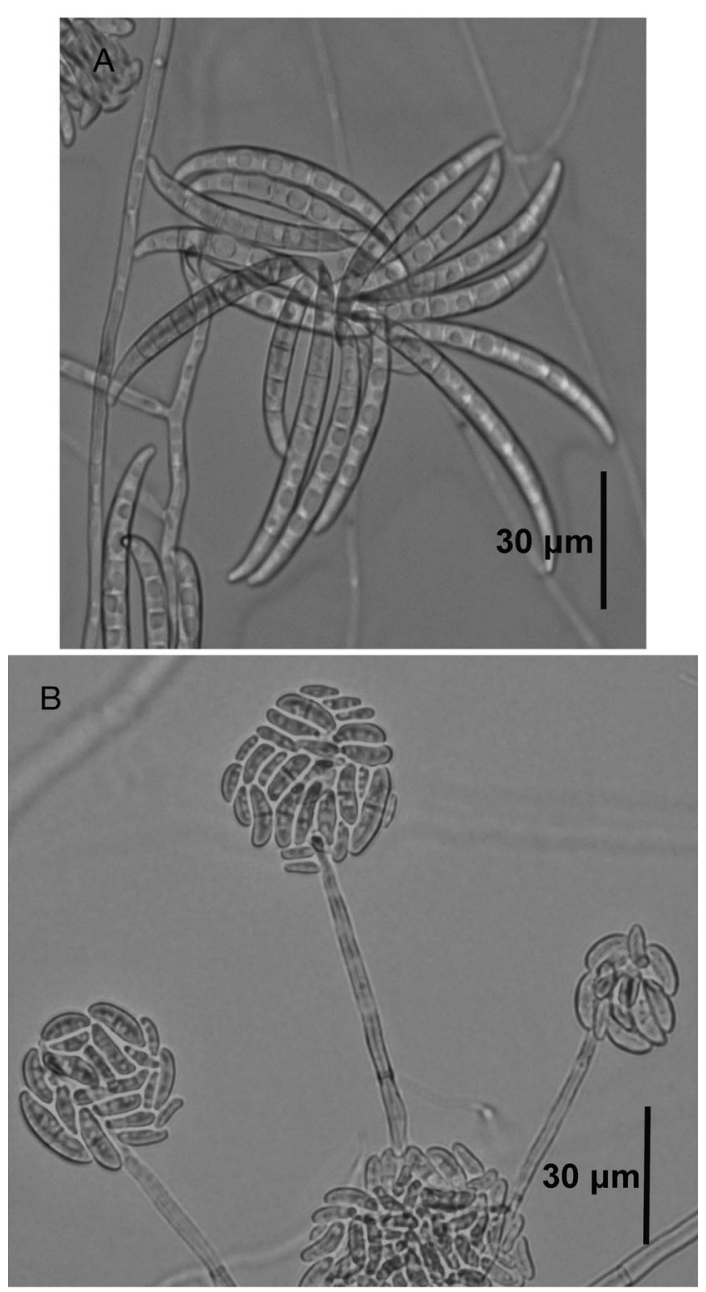

Fig. 4. Fusarium solani species complex (FSSC). Microscopic features of FSSC 12-a Fusarium sp. on carnation leaf agar after $7 \mathrm{~d}$ of incubation at $25^{\circ} \mathrm{C}$. Light micrographs of (A) fusiform, multiseptate macroconidia and (B) 1- and 2-celled microconidia formed in 'false heads' at the tips of long monophialides. Scale bars $=30 \mu \mathrm{m}$

conazole, and voriconazole resulted in minimum inhibitory concentrations (MIC) of $>64,>16,>16$, and $4 \mu \mathrm{g} \mathrm{ml}^{-1}$, respectively.

\section{DISCUSSION}

The similarity of lesions, as well as the consistent morphology of the hyphae observed in the seahorses, supports the conclusion that an FSSC 12-a Fusarium sp. was the etiologic agent in these cases. The distribution of lesions suggests that the fungus first established cutaneous infections, possibly in areas of previously damaged integument, and then spread hematogenously. Not all of the seahorses examined exhibited fungal invasion of internal organs, but 


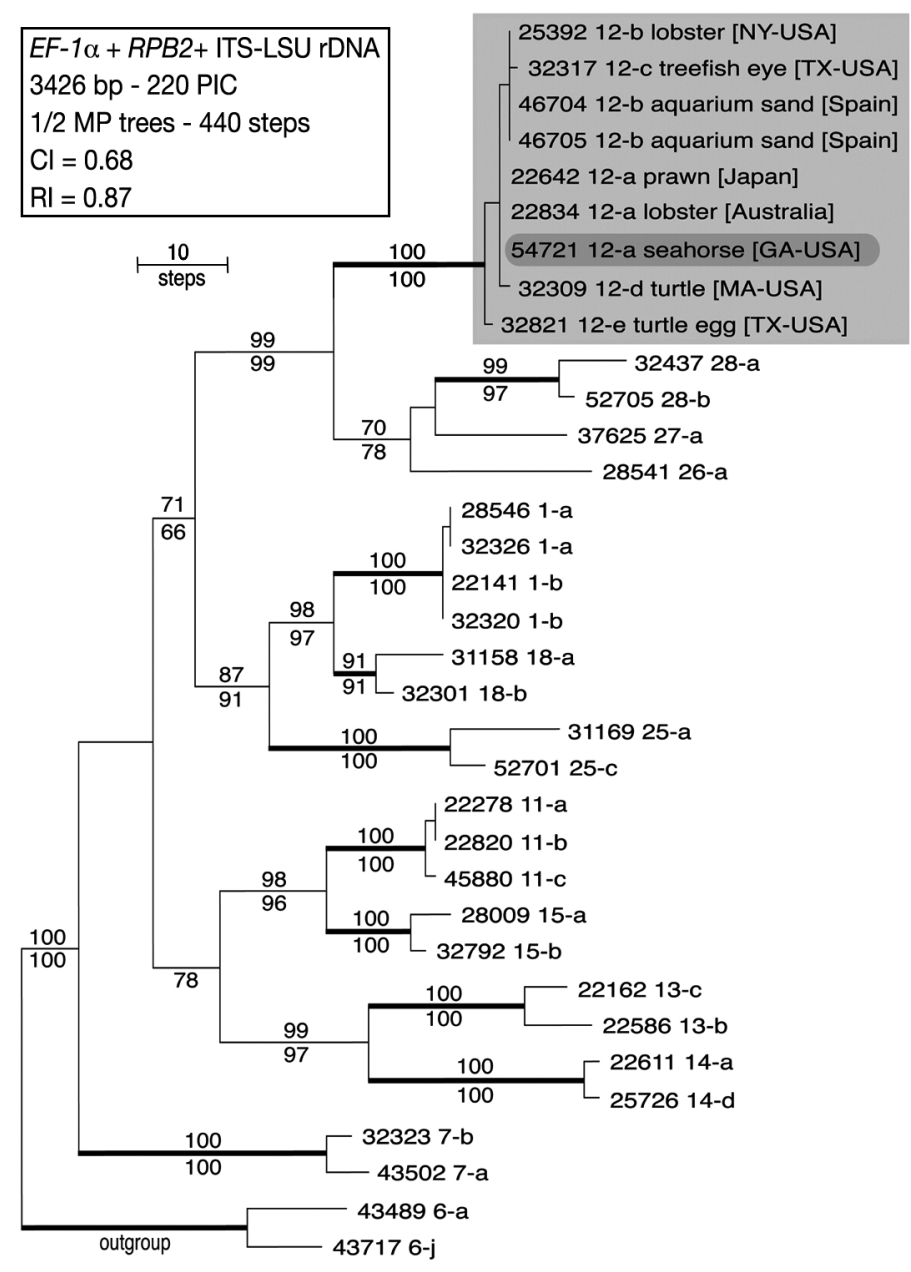

Fig. 5. One of 2 equally most-parsimonious phylograms inferred from the combined 3-locus dataset showing the phylogenetic relationship of the seahorse pathogen NRRL 54721 Fusarium solani species complex (FSSC) 12-a Fusarium sp. from Atlanta, GA, USA. Note that the 8 isolates of FSSC 12 (grey highlight) were all recovered from geographically diverse hosts/substrates in marine environments. Each of the 33 isolates in the dataset is identified by a 5-digit ARS Culture Collection accession number (NCAUR-ARS-USDA, Peoria, IL, USA), followed by an Arabic number identifying the phylogenetic species and lowercase Roman letter identifying the multilocus haplotype. Numbers above and below nodes represent, respectively, bootstrap support based on 1000 maximum parsimony (MP) and maximum likelihood (ML) pseudoreplicates of the data. Internodes that are thickened identify phylogenetic species represented by 2 or more isolates. The phylogram was rooted on sequences of FSSC 6 based on more inclusive analyses (O'Donnell et al. 2008). ITS-LSU rDNA: internal transcribed spacer region and nuclear large subunit; CI: consistency index; PIC: parsimony informative character; RI: retention index

these animals may have succumbed before systemic dissemination could occur. The fact that hyphae did not elicit overt necrosis and tissue damage in some of the seahorses may indicate that they succumbed to vascular thrombosis and infarction.
A potential role for bacteria, acting as either primary or secondary invaders, in these cases is unclear but cannot be ruled out. Bacteria were associated with skin surfaces, dermal tissues, and internal organs of multiple animals. However, as these seahorses were collected dead and several showed histologic evidence of autolysis, in the absence of corresponding tissue changes, their presence may represent post-mortem overgrowth. Bacteria are known to enter the integument and viscera of fish quickly after death (Noga 2010). In the single seahorse received unfixed, light growth of a Vibrio sp. Group 6 ( $V$. harveyi, $V$. alginolyticus, $V$. vulnificus, and $V$. parahaemolyticus) and Listonella (Vibrio) anguillarum were cultured from organs. Although bacteria were not observed in tissues of this animal, vibriosis, including ulcerative dermatitis, has been identified as a cause of high peracute to subacute mortalities in seahorses during post shipment acclimation (Koldewey 2005). In the present case, antibiotic treatments with oxolinic acid, trimethoprim sulfathiazole, and kanamycin were ineffective at stopping mortalities.

Granulomatous inflammation typical of fungal infection was not observed in these cases (Reimschuessel \& Ferguson 2006). While the lack of inflammation is uncharacteristic, it is not unprecedented. Systemic Exophiala sp. fungal infections in seadragons have also been reported to induce minimal inflammation. The authors postulated that immune responses in the seadragons may have been attenuated by stressors associated with captive husbandry (Nyaoke et al. 2009). Mortalities in these cases began 3 to $14 \mathrm{~d}$ following shipment, suggesting that immunosuppression secondary to transport may have predisposed the epizootic. Although its precise role in infections is unknown, production of the immunosuppressive compound cyclosporin A by members of the FSSC is another possible explanation for the limited inflammatory response. Cyclosporin A blocks transcription of human lymphokine genes in $\mathrm{T}$ lymphocytes and degranulation of granulocytes (Sugiura et al. 1999). Neither explanation, however, provides insight as to the source of the infection, although a common origin, such as food, water, or fomites, is suspected.

Treatment with itraconazole was initiated empirically based on histopathological findings, but failed to stop progression of the epizootic. Retrospective in vitro antifungal susceptibility testing on isolate NRRL 54721 FSSC 12-a Fusarium sp. demonstrated high MICs to all agents tested, including itraconaozle. While there are no CLSI-defined breakpoints for fusaria, even in humans, MICs beyond achievable serum concentrations using standard dosing regi- 
mens suggest a lack of clinical efficacy. These results are in accordance with published findings of MIC determinations performed on 10 antifungal compounds against 18 FSSC isolates that indicated limited in vitro efficacy against this group of fusaria and wide variability of MICs between species (Dignani \& Anaissie 2004).

A 3-locus DNA sequence-based typing scheme was used to identify the seahorse pathogen as a phylogenetically distinct species/haplotype within the FSSC, designated FSSC 12-a. In this informal nomenclatural scheme, species complex is indicated by upper case Roman letters, each species is identified by Arabic numerals, and lowercase Roman letters identify haplotypes within each species. This scheme was adopted within the FSSC and other veterinary and medically important species complexes within Fusarium (O'Donnell et al. 2009, 2010), because genealogical concordance phylogenetic species recognition (Taylor et al. 2000) has shown that these complexes contain many more species than formal names. In addition, the majority of Fusarium species, including most of the members of the FSSC, can only be identified definitively using DNA sequence data. Previous reports of FSSC 12 causing veterinary infections were reported as F. solani (Hatai et al. 1978, Rebell 1978) or Fusarium sp. (Lightner \& Fontaine 1975) based on morphological species recognition. Similar to other FSSC species pathogenic to animals, NRRL 54721 FSSC 12-a Fusarium sp. from seahorses is nested phylogenetically within Clade 3 of the FSSC. Virulence factors that allow species in Clade 3 to cause disease in animals are unknown. Proposed mechanisms include accelerated growth rates compared to other clade members, increased microconidia production, and the ability to grow at $37^{\circ} \mathrm{C}$ in mammals (Zhang et al. 2006, O'Donnell et al. 2008). However, water temperatures in the seahorse tanks were 9.5 to $13.5^{\circ} \mathrm{C}$ lower than $37^{\circ} \mathrm{C}$. Unlike other FSSC species, FSSC 12 isolates are from marine sources, including marine aquarium sediment and a number of marine animals, suggesting that they are adapted to marine habitats. Specifically, FSSC 12-a isolates have been implicated as a cause of mycosis in lobster, prawn (O'Donnell et al. 2008), and herein, the lined seahorse (Fig. 5).

Fusariosis has been reported sporadically as a cause of mortalities in fish and other aquatic organisms (Lightner \& Fontaine 1975, Hatai et al. 1978, Blazer \& Wolke 1979, Frasca et al. 1996, Cabañes et al. 1997, Yanong 2003, Noga 2010). Identification has often been limited to genus and based solely on morphologic features. The disease was devastating in this group of captive seahorses and has the potential to cause similar losses in other culture facilities, resulting in both economic losses and setbacks in species conservation. Accurate identification of fungal pathogens, particularly those within the genus Fusarium, is valuable for both epidemiological study and clinical management. Thus, our finding that FSSC 12 Fusarium sp. occupies a marine niche should be helpful in identifying its environmental reservoir and in preventing similar epizootics by screening fish and tanks for the presence of FSSC 12. Molecular typing of additional Fusarium isolates is warranted to allow further characterization of these species, thereby facilitating the development of improved diagnostics and treatment options for both human and veterinary patients.

Acknowledgements. We thank S. Sink for generating all DNA sequence data reported in this study and N. Orwig for collecting the DNA sequences in the National Center for Agricultural Utilization Research DNA core facility. Mention of firm names or trade products does not imply that they are endorsed or recommended by the US Department of Agriculture (USDA) over other firms or similar products not mentioned. The USDA is an equal opportunity provider and employer. Thanks also to E. H. Thompson and D. McCarthy of the Fungus Testing Laboratory for the morphologic workup of the isolate and antifungal susceptibility testing, respectively.

\section{LITERATURE CITED}

Ahnesjö I, Craig JF (2011) The biology of Syngnathidae: pipefishes, seadragons, and seahorses. J Fish Biol 78: 1597-1602

Allen TC (1992) Hematoxylin and eosin. In: Prophet EB, Mills B, Arrington JB, Sobin LH (eds) AFIP laboratory methods in histotechnology. American Registry of Pathology, Washington, DC, p 53-58

Azor M, Gené J, Cano J, Guarro J (2007) Universal in vitro antifungal resistance of genetic clades of the Fusarium solani species complex. Antimicrob Agents Chemother 51:1500-1503

> Bian BZ, Egusa S (1981) Histopathology of black gill disease caused by Fusarium solani (Martius) infection in the Kuruma prawn, Penaeus japonicus Bate. J Fish Dis 4: 195-201

Blazer VS, Wolke RE (1979) An Exophiala-like fungus as the cause of a systemic mycosis of marine fish. J Fish Dis 2: $145-152$

> Cabañes FJ, Alonso JM, Castellá G, Alegre F, Domingo M, Pont S (1997) Cutaneous hyalohyphomycosis caused by Fusarium solani in a loggerhead sea turtle (Caretta caretta L.). J Clin Microbiol 35:3343-3345

Chang DC, Grant GB, O'Donnell K, Wannemuehler KA and others (2006) Multistate outbreak of Fusarium keratitis associated with use of a new contact lens solution. JAMA 296:953-963

CLSI (Clinical and Laboratory Standards Institute) (2008) Reference method for broth dilution antifungal susceptibility testing of filamentous fungi. Approved standard 
M38-A2. Clinical and Laboratory Standards Institute, Wayne, PA

Dias TL, Rosa IL, Baum JK (2002) Threatened fishes of the world: Hippocampus erectus Perry, 1810 (Syngnathidae). Environ Biol Fishes 65:326

Dignani MC, Anaissie E (2004) Human fusariosis. Clin Microbiol Infect(Suppl 1):67-75

- Edgar RC (2004) MUSCLE: a multiple sequence alignment method with reduced time and space complexity. BMC Bioinformatics 5:113

Frasca S, Dunn JL, Cooke JC, Buck JD (1996) Mycotic dermatitis in an Atlantic white-sided dolphin, a pygmy sperm whale, and two harbor seals. J Am Vet Med Assoc 208:727-729

Geiser DM, del Mar Jiménez-Gasco M, Kang S, Makalowska I and others (2004) FUSARIUM-ID v. 1.0: a DNA sequence database for identifying Fusarium. Eur J Plant Pathol 110:473-479

Hatai K, Furuya K, Egusa S (1978) Studies on the pathogenic fungus associated with black gill disease of kuruma prawn, Penaeus japonicus. I. Isolation and identification of the BG-Fusarium. Fish Pathol 12:219-224

IUCN (International Union for Conservation of Nature) (2011) IUCN red list of threatened species. Version 2011.1. Available at www.iucnredlist.org

Khoa LV, Hatai K, Aoki T (2004) Fusarium incarnatum isolated from black tiger shrimp, Penaeus monodon Fabricius, with black gill disease cultured in Vietnam. J Fish Dis 27:507-515

Koldewey H (2005) Seahorse husbandry in public aquariums: 2005 Manual, with chapters contributed by members of the Syngnathid Discussion Group. ZSL, London

Kristensen HK (1948) An improved method of decalcification. Stain Technol 23:151-154

Lightner DV, Fontaine CT (1975) A mycosis of the American lobster, Homarus americanus, caused by Fusarium sp. J Invertebr Pathol 25:239-245

Mitchell JS, Attleburger MH (1973) Fusarium keratomycosis in the horse. Vet Med Small Anim Clin 68:1257-1260

Nelson PE, Toussoun TA, Marasas WFO (1983) Fusarium species. An illustrated manual for identification. Pennsylvania State University Press, University Park, PA

Nelson PE, Dignani MC, Anaissie EJ (1994) Taxonomy, biology, and clinical aspects of Fusarium species. Clin Microbiol Rev 7:479-504

Noga EJ (2010) Fish disease: diagnosis and treatment, 2nd edn. Wiley-Blackwell, Ames, IA

Nyaoke A, Weber ES, Innis C, Stremme D and others (2009) Disseminated phaeohyphomycosis in weedy seadragons (Phyllopteryx taeniolatus) and leafy seadragons (Phycodurus eques) caused by species of Exophiala, including a novel species. J Vet Diagn Invest 21:69-79

O'Donnell K (2000) Molecular phylogeny of the Nectria haematococca - Fusarium solani species complex. Mycologia 92:919-938

O'Donnell K, Cigelnik E, Nirenberg H (1998) Molecular systematic and phylogeography of the Gibberella fujikuroi species complex. Mycologia 90:465-493

O'Donnell K, Sutton DA, Fothergill A, McCarthy D and others (2008) Molecular phylogenetic diversity, multilocus haplotype nomenclature, and in vitro antifungal resistance within the Fusarium solani species complex. J Clin Microbiol 46:2477-2490
O'Donnell K, Sutton DA, Rinaldi MG, Crous PW, Geiser DM (2009) A novel MLST scheme reveals high genetic diversity of human pathogenic members of the Fusarium incarnatum-equiseti and F. chlamydosporum species complexes within the U.S. J Clin Microbiol 47: 3851-3861

O'Donnell K, Sutton DA, Rinaldi MG, Sarver BAJ and others (2010) Internet-accessible DNA sequence database for identifying fusaria from human and animal infections. J Clin Microbiol 48:3708-3718

Palmero D, Iglesias C, de Cara M, Lomas T, Santos M, Tello JC (2009) Species of Fusarium isolated from river and sea water of southeastern Spain and pathogenicity on four plant species. Plant Dis 93:377-385

> Perpiñán D, Trupkiewicz JG, Armbrust AL, Geiser DM, Armstrong S, Garner MM, Armstrong DL (2010) Dermatitis in captive Wyoming toads (Bufo baxteri) associated with Fusarium spp. J Wildl Dis 46:1185-1195

Rebell G (1978) Fusarium infections in human and veterinary medicine. In: Nelson PE, Toussoun TA, Cook RJ (eds) Fusarium: diseases, biology, and taxonomy. Pennsylvania State University Press, University Park, PA, p 210-220

Reimschuessel R, Ferguson HW (2006) Kidney. In: Ferguson HW (ed) Systemic pathology of fish. Scotian Press, London, p 91-118

Rinaldi MG (1982) Use of potato flakes agar in clinical mycology. J Clin Microbiol 15:1159-1160

Roilides E, Dotis J, Katragkou A (2007) Fusarium and Scedosporium: emerging fungal pathogens. In: Kavanagh K (ed) New insights in medical mycology. Springer, Dordrecht, p 267-286

Sarmiento-Ramírez JM, Abella E, Martín MP, Tellería MT, López-Jurado LF, Marco A, Diéguez-Uribeondo J (2010) Fusarium solani is responsible for mass mortalities in nests of loggerhead sea turtle, Caretta caretta, in Boavista, Cape Verde. FEMS Microbiol Lett 312:192-200

Stoskopf MK (1993) Clinical examination and procedures. In: Stoskopf MK (ed) Fish medicine. WB Saunders, Philadelphia, PA, p 62-78

> Sugiura Y, Barr JR, Barr DB, Brock JW and others (1999) Physiological characteristics and mycotoxins of human clinical isolates of Fusarium species. Mycol Res 103: 1462-1468

Swofford DL (2002) PAUP*. Phylogenetic analysis using parsimony ( ${ }^{*}$ and other methods), version 4 . Sinauer Associates, Sunderland, MA

Taylor JW, Jacobson DJ, Kroken S, Kasuga T, Geiser DM, Hibbett DS, Fisher MC (2000) Phylogenetic species recognition and species concepts in fungi. Fungal Genet Biol 31:21-32

> Vincent ACJ, Foster SJ, Koldewey HJ (2011) Conservation and management of seahorses and other Syngnathidae. J Fish Biol 78:1681-1724

Yanong RPE (2003) Fungal diseases of fish. Vet Clin North Am Exot Anim Pract 6:377-400

Z Zhang N, O'Donnell K, Sutton DA, Nalim FA, Summerbell RC, Padhye AA, Geiser DM (2006) Members of the Fusarium solani species complex that cause infections in both humans and plants are common in the environment. J Clin Microbiol 44:2186-2190

Zwickl DJ (2006) Genetic algorithm approaches for the phylogenetic analysis of large biological sequence data sets under the maximum likelihood criterion. $\mathrm{PhD}$ dissertation, University of Texas, Austin, TX 